\title{
Bladder leiomyoma - an unusual cause of acute urinary retention
}

\begin{abstract}
Introduction: Bladder leiomyomas are benign mesenchymal neoplasms with only 250 cases reported worldwide to date. They can be asymptomatic, but the majority present with obstructive symptoms (49\%), irritative symptoms $(38 \%)$ and hematuria $(11 \%)$. The importance of recognizing their characteristic features, leading to their correct treatment, is fundamental.
\end{abstract}

Methods: Case report and review of pertinent English medical literature.

Results: A 53 year old lady presented with urgency and frequency for 3 months, with two episodes of painless total hematuria. She was catheterized for acute urinary retention elsewhere. Clinical examination was unremarkable. Ultrasound (US) showed a heterogeneously hypoechoic lesion of $4 \times 3 \mathrm{~cm}$ in bladder base. Contrast enhanced computed tomogram (CECT) showed a $5 \times 4 \mathrm{~cm}$ well circumscribed hypodense lesion arising from trigone, without hydroureteronephrosis. A mucosal bulge measuring around $5 \times 5 \mathrm{~cm}$ was seen in the right lateral wall extending upto bladder neck at cystoscopy. Transurethral resection (TUR) was done and biopsy showed fragments of tumor tissue composed of spindle cells arranged in sheets and interlacing fascicles, without mitosis or necrosis, suggestive of leiomyoma bladder. Immunohistochemistry showed SMA and desmin positivity. Complete TUR enuceation was done and patient is doing well at 6 months of follow up without recurrence or hematuria.

Conclusion: Leiomyoma of the bladder should be kept in mind in chronic lower urinary tract symptoms with acute urinary retention and hematuria in patients presenting in the third to sixth decade, especially in women. A complete assessment to look for endovesical leiomyoma should be made.

Keywords: leiomyoma, endovesical, acute urinary retention, hematuria, transurethral resection
Volume 8 Issue 4 - 2017

\author{
Sujatha Siddappa,Vijay Ganapathy S, Vinay \\ Saini A, Keshavamurthy R \\ Department of Urology, Institute of Nephro Urology, India
}

Correspondence: Sujatha Siddappa, Department of Urology, Institute of Nephro Urology, India, Tel (2I5)728-3850, Fax (215)728-2848,Email sujathasiddappa@gmail.com

Received: January 27, 2017 | Published: September 6, 2017

\section{Introduction}

Bladder leiomyomas are benign mesenchymal neoplasms and very rare urinary tumours that represent $<0.5 \%$ of all bladder tumours, with only 250 cases reported worldwide to date..$^{1,2}$. Patients with bladder leiomyomas can be asymptomatic, but the majority present with obstructive symptoms (49\%), irritative symptoms (38\%) and hematuria $(11 \%))^{3,4}$ The importance of recognizing their characteristic features, leading to their correct treatment, is fundamental.

\section{Case details}

A 53year old lady presented with urgency and frequency for 3 months, with two episodes of painless total gross hematuria. She developed acute urinary retention and was catheterized elsewhere and referred to our institute. She had no urological or spine surgeries, no known comorbidities or prior episodes of hematuria. Clinical examination was unremarkable. Ultrasound (US) showed a heterogeneously hypoechoic lesion of $4 \times 3 \times 4 \mathrm{~cm}$ in bladder base (Figure 1). Contrast enhanced computed tomogram (CECT) showed a $5 \times 4 \times 4 \mathrm{~cm}$ well circumscribed hypodense lesion arising from trigone, without hydroureteronephrosis (Figure 2). At cystoscopy, a mucosal bulge measuring around $5 \times 5 \mathrm{~cm}$ was seen in the right lateral wall extending upto bladder neck and a cecoureterocele was suspected (Figure 3). Transurethral resection (TUR) was done and it showed fragments of tumor tissue composed of spindle cells arranged in sheets and interlacing fascicles, without mitosis or necrosis, suggestive of leiomyoma bladder (Figure 4). Immunohistochemistry showed smooth muscle antigen (SMA) and desmin positivity (Figure 5). Repeat TUR and complete enuceation of the lesion was done. At 6 months of follow up patient is doing well without recurrence or hematuria.

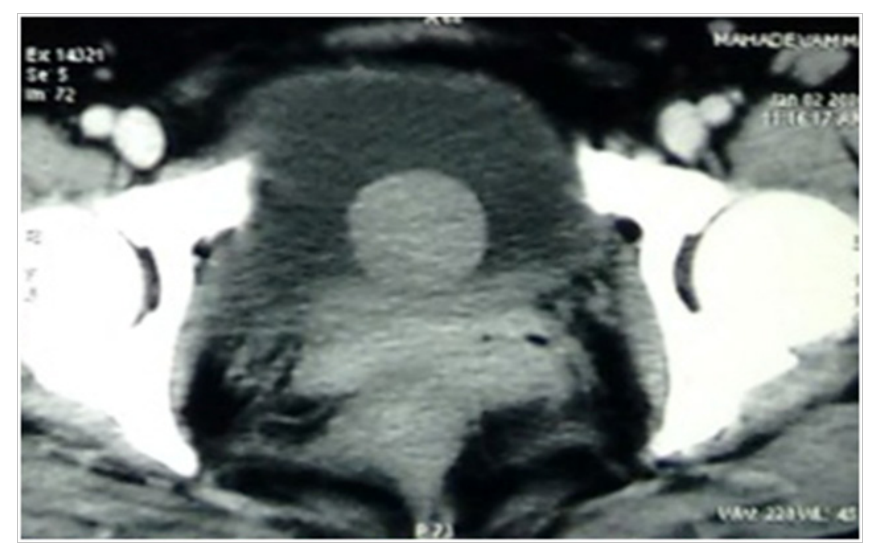

Figure I Ultrasound (US) showed a heterogeneously hypoechoic lesion of 4 $\times 3 \times 4 \mathrm{~cm}$ in bladder base.

\section{Discussion}

Leiomyoma is a benign mesothelial tumor and consists of four different subtypes in the bladder. ${ }^{5}$. Epitheloid subtype is the rarest 
type. Leiomyomas are classified into three different types based on location - endovesical, intramural and extravesical.6Endovesical leiomyoma is the most common location, in $63-86 \%$ of cases. Intramural leiomyomas present in $3-7 \%$ and extravesical location in $11-30 \%$, with female preponderance $(70 \%)$ affecting patients in their third to sixth decades. ${ }^{3-7}$ The most common symptoms at are obstructive (49\%), followed by irritative (38\%) and hematuria (11\%).

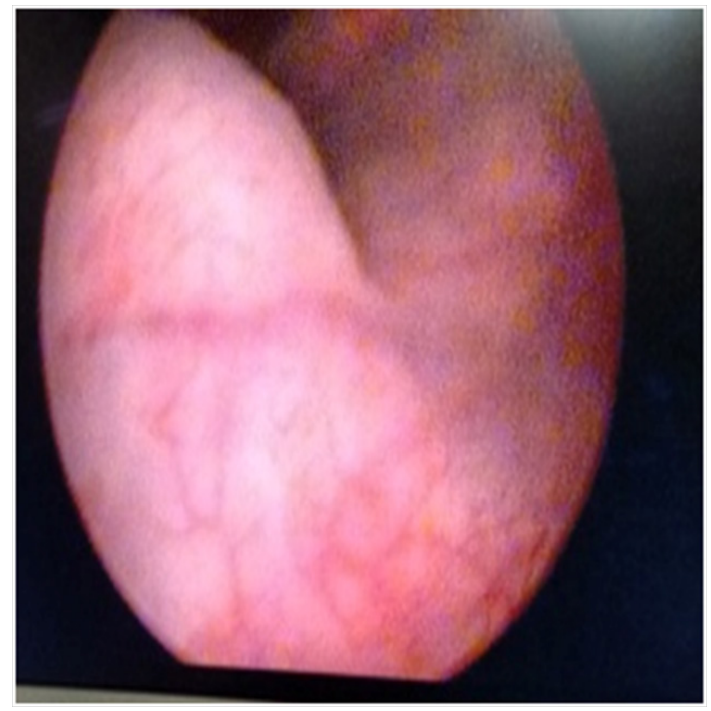

Figure 2 Contrast enhanced computed tomogram (CECT) showed a $5 \times 4$ $\times 4 \mathrm{~cm}$ well circumscribed hypodense lesion arising from trigone, without hydroureteronephrosis.

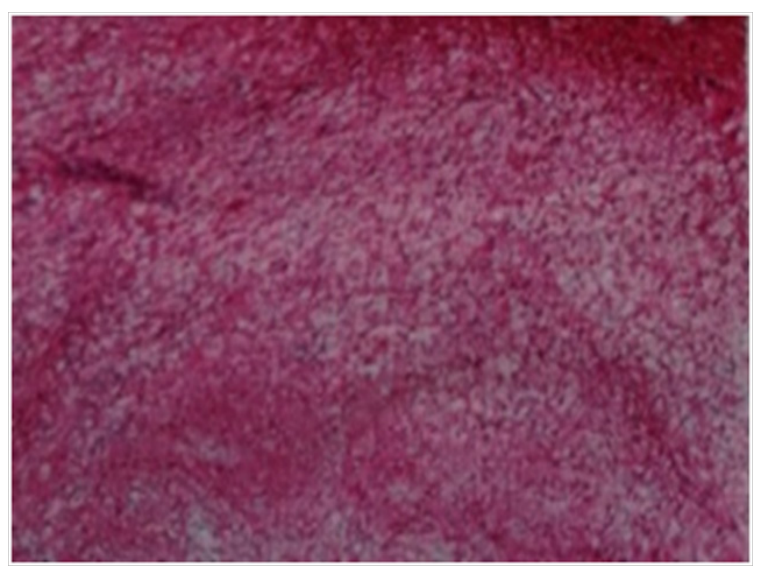

Figure 3 Cystoscopy - a mucosal bulge measuring around $5 \times 5 \mathrm{~cm}$ was seen in the right lateral wall extending upto bladder neck.

Leiomyomas of the urinary bladder share common histopathological features with leiomyomas of the uterus, characterized by grey-white round nodules with a spiral appearance of smooth muscle fibres gathered in small fascicles and separated by varying amounts of fibrous connective tissue, and fewer than two mitotic figures per highpower field. ${ }^{3,5,8}$ The endovesical subtype is more common because it bulges into the bladder, resulting in more symptoms and causing the patient to seek medical attention. As the endovesical location is the most common, TUR represents the main treatment in almost $90 \%$ of cases. ${ }^{9} 10$ A large intramural or an extravesical leiomyoma requires wide excision or partial cystectomy. Surgical treatment is almost always very effective, leaving a very low recurrence rate, and with no symptoms, confirming the benign nature of bladder leiomyomas and their excellent prognosis. ${ }^{11,12}$

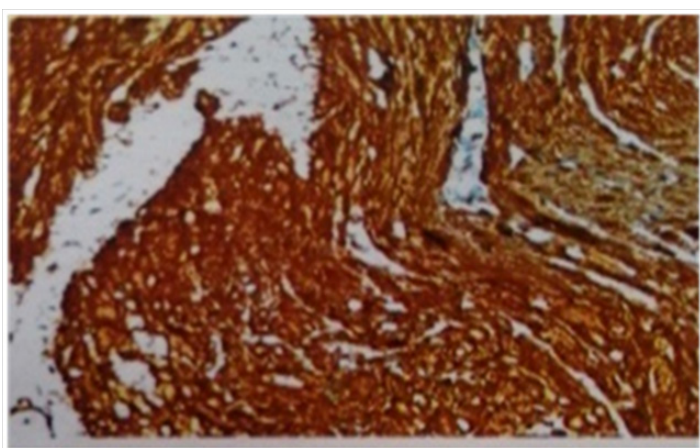

Figure 4 Photomicrograph. Leiomyoma - spindle cells arranged in sheets and interlacing fascicles, without mitosis or necrosis H\&E, 40X).

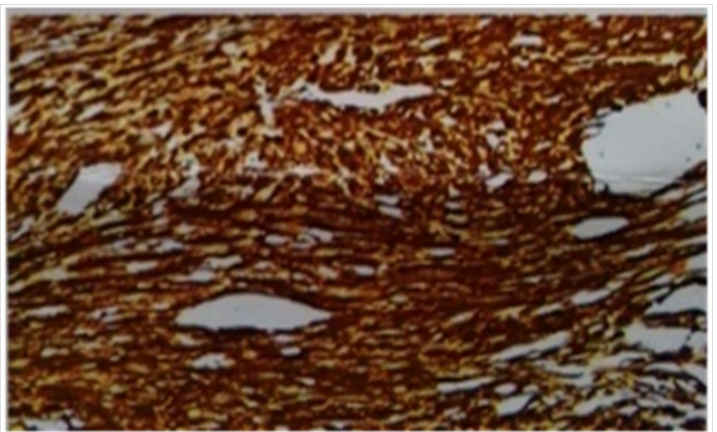

Figure 5 Photomicrograph. Immunohistochemistry showing smooth muscle antigen (SMA) and desmin positivity (40X).

A better understanding of this disease in the future might perhaps offer a new intravesical agent that will target these cells specifically. Although urinary cytology studies are being used as options for assessing transitional cell carcinoma of the urinary tract, they might be developed as new methods that could be applied to bladder leiomyoma (although benign), for screening, follow-up and perhaps to avoid the sometimes unnecessary aggressive measures.

\section{Conclusion}

Leiomyoma of the bladder is an uncommon neoplasm. It should be kept in mind in chronic lower urinary tract symptoms with acute urinary retention and hematuria in patients presenting in the third to sixth decade, especially in women. A complete assessment to look for endovesical leiomyoma should be made.

\section{Acknowledgements}

None.

\section{Conflicts of interest}

The authors declare that there are no conflicts of interest.

\section{Funding}

None.

\section{References}

1. Campbell EW, Gislason GJ. Benign mesothelial tumors of the urinary bladder: review of literature and a report of a case of leiomyoma. J Urol. 1953;70(5):733-742. 
2. Cornella JL, Larson TR, Lee RA, et al. Leiomyoma of the female urethra and bladder: Report of twenty three patients and review of literature. $\mathrm{Am}$ J Obstet Gynecol. 1997;176(6):1278-1285.

3. Goluboff ET, O'Toole K, Sawczuk IS. Leiomyoma of the bladder: Report of a case and review of the literature. Urology. 1994;43:238-241.

4. Saunders SE, Conjeski JM, Zaslau S, et al. Leiomyoma of the urinary bladder presenting as urinary retention in the female. Can $J$ Urol. 2009;16(4):4762-4764.

5. Knoll LD, Segura JW, Scheithauer BW. Leiomyoma of the bladder. $J$ Urol. 1986;136(4):906-908.

6. Park JW, Jeong BC, Seo SI, et al. Leiomyoma of the urinary bladder: A series of nine cases and review of the literature. Urology. 2010;76(6):1425-1429.
7. Illescas FF, Baker ME, Weinerth JL. Bladder leiomyoma. Advantages of sonography over computed tomography. Urol Radiol. 1986;8(4):216218 .

8. Silva-Ramos M, Massó P, Versos R, et al. Leiomyoma of the bladder. Analysis of a collection of 90 cases. Actas Urol Esp. 2003;27:581-586.

9. Erdem H, Yildirim U, Tekin A, et al. Leiomyoma of the urinary bladder. Urology Annals. 2012;4(3):172-174.

10. Jeschke K, Wakonig J, Winzely M, et al. Laparoscopic partial cystectomy for leiomyoma of the bladder wall. J Urol. 2002;168(5):2115-2116.

11. Lake MH, Kossow AS, Bokinsky G. Leiomyoma of the bladder and urethra. J Urol. 1981;125(5):742-743

12. Soloway D, Simon MA, Milikowski C, et al. Epithelioid leiomyoma of the bladder: An unusual cause of voiding symptoms. Urology. 1998;51(6):1037-1039. 